\title{
A Workflow for Imaging 2D Materials using 4D STEM-in-SEM
}

\author{
Benjamin W. Caplins ${ }^{1 *}$, Ryan M. White ${ }^{1}$, Jason D. Holm ${ }^{1}$ and Robert R. Keller ${ }^{1}$ \\ 1. Material Measurement Lab, National Institute of Standards and Technology, Boulder, CO, USA \\ * Corresponding author: benjamin.caplins@ @ist.gov
}

The grain structure and orientation of polycrystalline two-dimensional (2D) materials modulates their thermal, mechanical, and electrical properties. Thus, to rationally control the properties of 2D materials, the as-synthesized grain structure of the 2D materials must be routinely and reliably measured. Although different methods can be used to spatially map the grain structure/orientation of 2D materials, electron diffraction methods are generally regarded as the 'ground truth' because they can resolve these characteristics on the nanometer length scale.

In this contribution, a four-dimensional (real-space and k-space) scanning transmission electron microscopy method for use in the scanning electron microscope (4D STEM-in-SEM) is described. This technique can map the structure/orientation of $2 \mathrm{D}$ materials on a wide range of length scales $\left(10^{-2} \mathrm{~m}-\right.$ $\left.10^{-9} \mathrm{~m}\right)$. Briefly, a $30 \mathrm{keV}$ focused electron beam is incident on the electron transparent 2D material. The electrons that transmit through the sample propagate in a field-free region and strike a phosphor screen forming a diffraction pattern. This (optical) diffraction pattern is then imaged onto a CCD camera. A home-built scan generator is used to raster the electron beam across the sample and trigger the storage of diffraction pattern images [1]. The low energy of the electron beam yields significant diffraction contrast while creating minimal knock-on damage [2].

Once collected, the output of a 4D STEM-in-SEM experiment can be regarded as a 'big' dataset which cannot be exhaustively inspected by humans - e.g., a single real-space scan $(512 \times 512)$ generates $\sim 250000$ diffraction patterns. We have developed a workflow based on a Fourier-space representation of the diffraction data to rapidly inspect 4D STEM-in-SEM data and extract information such as crystallographic orientation and identify distinct regions of the material (such as different thicknesses and interlayer rotations). A typical workflow for data inspection is as follows. First, since no de-scan coils exist in an SEM, the center of each diffraction pattern is located and de-scanned digitally. This step is particularly important for large fields-of-view. The diffraction pattern is then resampled to a polar grid, and the Fourier transform is applied to the angular coordinate. The transformed data can be visualized by creating colorized image of the amplitude and phase of the Fourier-space data. Since it can be reasonably assumed that the diffraction patterns of a $2 \mathrm{D}$ material are based on structures that have in-plane symmetry, we can initially restrict our inspection to just a few low-index Fourier components. These synthetic images allow a practical visualization of the 4D STEM-in-SEM dataset with minimal processing power and user time and can indicate what region/structures are present in the sample. Then a cross-correlation-based pattern matching approach can give higher quality orientation information. This Fourier representation may be a useful dimensionality reduction method when machine learning is used for further analysis.

Figure 1 shows examples of this Fourier based analysis applied to a graphene oxide sample. An image of the phase of a six-fold Fourier component is used to visualize the grain orientation, and the amplitude image to visualize multilayer structures. Figure 2 shows the technique applied to monolayer graphene. Inspection of the two-fold Fourier components unexpectedly reveals texture on the micrometer length 
scale that was assigned as polymer residue from a wet-transfer step. Furthermore, the analysis showed that the polymer was oriented with respect to the graphene lattice [3]. The workflow described here leverages the symmetry of 2D materials and the efficiency of the FFT and may be particularly helpful when the S/N and processing power is not available/necessary to perform a more detailed analysis [4].

\section{References:}

[1] W.C. Lenthe et al., Ultramicroscopy 195 (2018), p. 93.

[2] R.F. Egerton, Micron 119 (2019), p. 72.

[3] M. Gulde et al., Science 345 (2014), p. 200.

[4] Y. Han et al., Nano Letters 18 (2018), p. 3746.

[5] This work is a contribution of the US Government and is not subject to United States copyright.

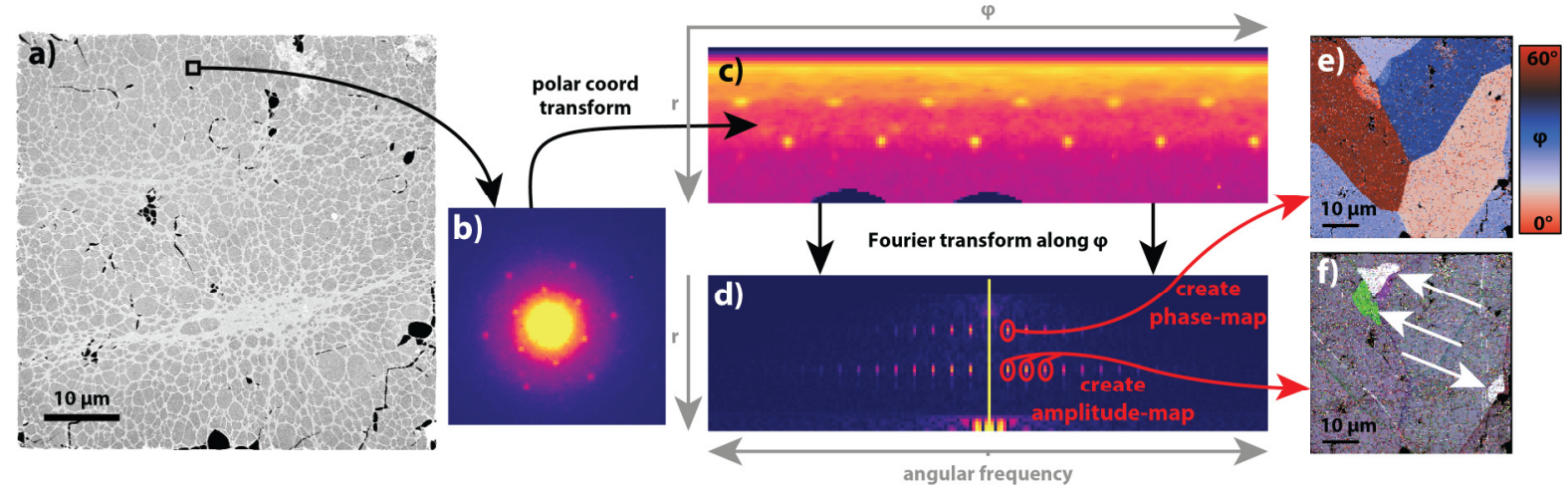

Figure 1. a) Secondary electron image of a mostly monolayer graphene oxide film on lacey carbon. b) A single diffraction pattern from a 4D STEM-in-SEM dataset. c) Diffraction pattern resampled to polar coordinates. d) Absolute value of the FFT (across $\varphi$ ) of the polar data. e) Colorized map of the phase of the circled Fourier component. f) Colorized map of the amplitudes of the circled Fourier components; distinct bilayer regions indicated with arrows.
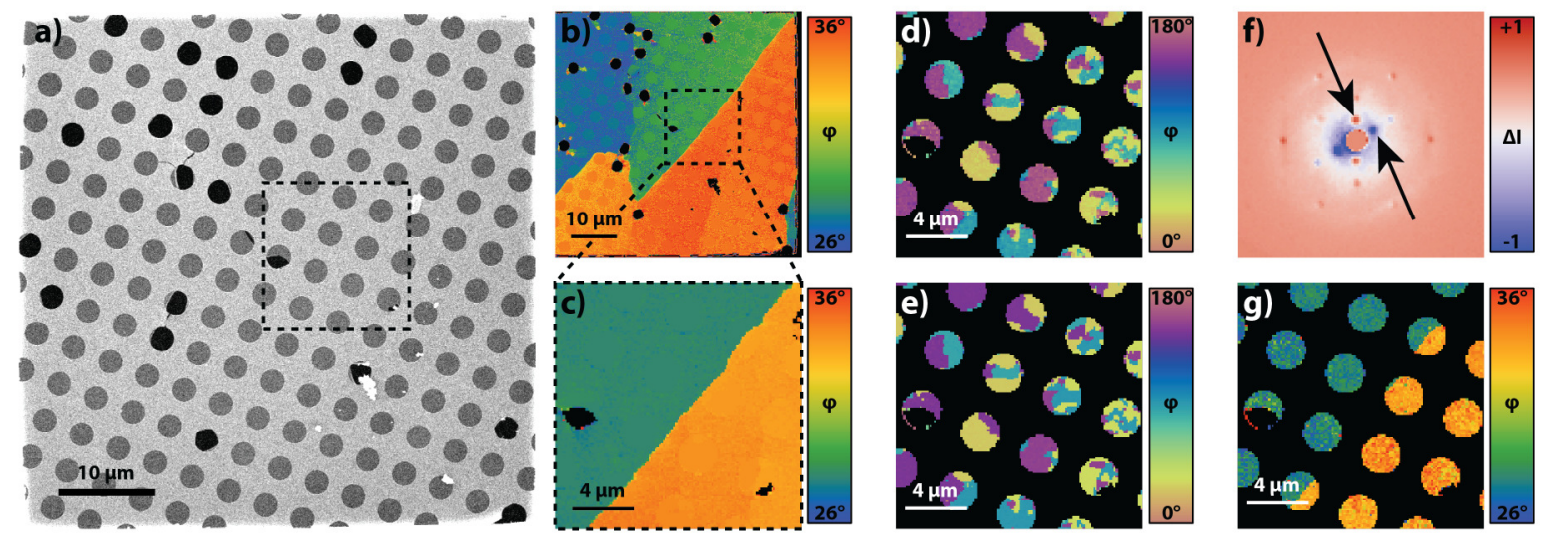

Figure 2. a) Secondary electron image of a supported monolayer graphene film. b) Colorized map of the phase of a six-fold Fourier component - i.e., graphene orientation. c) Zoomed-in orientation map derived using a cross-correlation approach. d) Colorized map of the phase of a two-fold Fourier component showing the texture of a polymer residue. e) Orientation map using a cross-correlation approach. f) The difference between the diffraction patterns in two polymer domains; the arrows indicate the polymer diffraction. g) The data in (e) modulo $60^{\circ}$, on the same colormap as (c), highlighting the orientation relationship. 